\title{
Vitamin D Studies: Mistaking Correlation for Causation
}

\author{
Seung-Won Oh \\ Department of Family Medicine, Healthcare System Gangnam Center, Seoul National University Hospital, Seoul, Korea
}

There has been a growing interest in the study of vitamin D. Numerous conference presentations on vitamin D and related articles can be easily found through media outlets or on the internet. In a doctor's office, it is also easy to meet patients who have taken or have been injected with vitamin D. Levels of vitamin $\mathrm{D}$, unlike other vitamins, can be tested easily via a blood test; hence, testing has also been on the rise. Despite controversy related to optimal vitamin $\mathrm{D}$ level, most experts define vitamin D insufficiency as less than $20 \mathrm{ng} / \mathrm{mL}$ of 25 -hydroxyvitamin $\mathrm{D}[25(\mathrm{OH}) \mathrm{D}]$. $^{1)}$

These phenomena are based on recently reported vitamin D study results. Epidemiological studies suggest that vitamin $\mathrm{D}$ insufficiency is a common issue in Korea and worldwide, bringing attention to vitamin D insufficiency. In the study based on the Fourth Korea National Health and Nutrition Examination Surveys (KNHANES IV) conducted in 2008, vitamin D insufficiency was found in $47.3 \%$ of men and $64.5 \%$ of women. ${ }^{2)}$ A recent study of over 50,000 Europeans also found about $40 \%$ prevalence of vitamin D insufficiency. ${ }^{3)}$ In addition, there have been reports that suggest that vitamin $\mathrm{D}$ is correlated not only with the musculoskeletal system, but also with various diseases including cancer and cardiovascular diseases, by which elevation of vitamin $\mathrm{D}$ level in the body becomes a global issue that goes beyond mere interest.

Thousands of studies on vitamin D have been reported every year, and the number of related studies tends to increase continuously. These studies often include not only diseases with relatively higher number of studies such as cancer, cardiovascular diseases, and diabetes, but also infectious diseases such as tuberculosis, autoimmune diseases, and mental diseases such as depression. Most of these studies report on the correlation of $25(\mathrm{OH})$ vitamin D level with disease risk.

In the present issue, Kim et al. examined the relationship between vitamin D status and health-related quality of life us- ing KNHANES. ${ }^{4)}$ In interpreting vitamin D-related study results, correlation should not be understood as causation. Diets composed of vitamin D-rich foods such as dairy products and salmon also contain high levels of other healthy nutrients. Those who have a high vitamin D level are likely to participate in active outdoor activities and exercises, to be interested in health issues, and to have a healthy lifestyle. Without considering these confounders, misleading results can be obtained. In the study by Kim et al., ${ }^{4)}$ a univariate analysis revealed a correlation between a low vitamin $\mathrm{D}$ level and a low quality of life score; however, its significance was lost when age, sex, income, education level, and disease state were considered.

Sometimes, correlations shown in cross-sectional studies are used as evidence for requiring vitamin D supplements. A recent increasing trend of taking vitamin D supplements may be due to these effects. Previous studies such as those that implied increased risk of lung cancer in smokers via increased $\beta$-carotene intake indicate that taking a vitamin supplement may be unexpectedly harmful to a person's health. ${ }^{5)}$ Although causation for a certain disease is identified through observational studies, reduction of the corresponding disease incidence through supplementation is another issue. To date, there is no foundation for recommending that healthy people should take vitamin D supplements. At present, there are large-scale randomized controlled clinical trials studying the effects of vitamin D supplementation on cancer, cardiovascular diseases, and mortality, and people should be mindful of their use of vitamin D supplements until a firm foundation is established through the research. ${ }^{6}$

\section{CONFLICT OF INTEREST}

No potential conflict of interest relevant to this article was reported. 


\section{REFERENCES}

1. Rosen CJ. Clinical practice: vitamin D insufficiency. N Engl J Med 2011;364:248-54.

2. Choi HS, Oh HJ, Choi H, Choi WH, Kim JG, Kim KM, et al. Vitamin D insufficiency in Korea: a greater threat to younger generation: the Korea National Health and Nutrition Examination Survey (KNHANES) 2008. J Clin Endocrinol Metab 2011;96:643-51.

3. Cashman KD, Dowling KG, Skrabakova Z, Gonzalez-Gross M, Valtuena J, De Henauw S, et al. Vitamin D deficiency in Europe: pandemic?
Am J Clin Nutr 2016;103:1033-44.

4. Kim JS, Choi YE, Baek JK, Cho HJ, Kim YS. The association between vitamin D and health-related quality of life in Korean adults. Korean J Fam Med 2016;37:221-7.

5. The Alpha-Tocopherol, Beta Carotene Cancer Prevention Study Group. The effect of vitamin $\mathrm{E}$ and beta carotene on the incidence of lung cancer and other cancers in male smokers. N Engl J Med 1994;330:1029-35.

6. Manson JE, Bassuk SS. Vitamin D research and clinical practice: at a crossroads. JAMA 2015;313:1311-2. 